Check for updates

Cite this: RSC Adv., 2019, 9, 8315

Received 9th December 2018

Accepted 4th March 2019

DOI: $10.1039 / c 8 r a 10103 e$

rsc.li/rsc-advances

\section{Enhancement of neural stem cell survival, proliferation and differentiation by IGF-1 delivery in graphene oxide-incorporated PLGA electrospun nanofibrous mats $\dagger$}

\author{
Zhiping Qi, ${ }^{a}$ Wenlai Guo, ${ }^{a}$ Shuang Zheng, ${ }^{a}$ Chuan Fu, ${ }^{a}$ Yue Ma, ${ }^{\text {b }}$ Su Pan, ${ }^{a}$ Qinyi Liu*a \\ and Xiaoyu Yang*a
}

The mammalian central nervous system has a limited ability for self-repair under injury conditions. The treatment of nerve injuries has been revolutionised with the development of tissue engineering techniques. However, the lack of bioactivity has severely restricted the application of biodegradable implants for neurogenesis. Therefore, surface modification of biomaterials is crucial to improve their bioactivity and promote endogenous repair mechanisms for nerve regeneration. Insulin-like growth factor 1 (IGF-1) is a growth factor for neuroprotection and neurogenesis. In this study, IGF-1 was successfully immobilised on graphene oxide (GO)-incorporated poly(lactic-co-glycolic acid) (PLGA) biodegradable electrospun nanofibres. For the in vitro investigation, neural stem cells (NSCs) were cultured on different nanofibres to observe various cellular activities. GO enhanced NSC survival under $\mathrm{H}_{2} \mathrm{O}_{2}$ pre-treatment and neuronal differentiation to some extent. More importantly, the immobilisation of IGF-1 onto the PLGA/GO nanofibres resulted in significantly increased NSC survival, proliferation, and differentiation. Findings from this study revealed that using PLGA/GO electrospun nanofibres to immobilise IGF-1 has excellent potential for the enhancement of the neuroprotective and neurogenic effects of nerve implants.

\section{Introduction}

Nerve injury is a common global health problem that can significantly affect the quality of life of patients and remains one of the most challenging clinical problems. ${ }^{1}$ Although endogenous neural stem cells (NSCs) are immediately activated after nerve injury and migrate towards the lesioned site, the hostile pathological microenvironment is unfavourable for endogenous NSC survival, proliferation and differentiation due to inflammation, reactive oxygen species (ROS) overproduction, cystic cavitation, and the presence of growth-inhibiting molecules in myelin. ${ }^{2-4}$ Therefore, the use of biomaterial scaffolds and growth factor combinations could be a promising strategy for nerve repair and regeneration.,

Biodegradable polymers have been widely used to prepare tissue engineering scaffolds due to their versatile properties. Synthetic biodegradable polymers, such as poly(lactic-co-

${ }^{a}$ Department of Orthopedic Surgery, The Second Hospital of Jilin University, Ziqiang Street No. 218, Changchun TX 130041, Jilin, PR China. E-mail: qinyi@jlu.edu.cn; yangxiaoy@jlu.edu.cn

${ }^{b}$ Department of Gynecological Oncology, The First Hospital of Jilin University, Xinmin Street No. 71, Changchun TX 130000, PR China

$\dagger$ Electronic supplementary information (ESI) available. See DOI: 10.1039/c8ra10103e glycolic acid) (PLGA), alone or combined with other active factors have been extensively studied for tissue engineering because these polymers possess excellent mechanical properties, low immunogenicity and toxicity, and an adjustable degradation rate. ${ }^{7,8}$ However, the hydrophobic surfaces of PLGA do not promote cell adhesion, and PLGA does not have bioactivity on neurogenesis. One strategy to overcome this problem is surface modification by incorporating cell growth factors into the scaffolds. There are various growth factors, such as insulinlike growth factor 1 (IGF-1), epidermal growth factor (EGF) and neural growth factor (NGF), that are associated with neurogenesis. ${ }^{9-11}$ IGF-I influences a wide range of physiological functions, including growth, differentiation, and metabolism. IGF-1 is a mitogenic factor that substantially enhances NSC proliferation both in vitro and in vivo. ${ }^{12,13}$ A previous study has shown that IGF-1 promotes the proliferation of NSCs by interacting with the insulin-like growth factor 1 receptor (IGF-1R), which may activate the PI3K/AKT and MAP kinase pathways. ${ }^{14}$ The IGF/IGFR system regulates the differentiation of neurons, astrocytes, and oligodendrocytes generated from NSCs in embryonic and adult brains. ${ }^{15,16}$ In addition, IGF is also involved in the regulation of NSC migration, which is mediated by the activation of the PI3K pathway and by phosphorylation of the reelin signal transducer. ${ }^{17}$ Previous studies have also 
demonstrated that IGF-I promotes cell survival by inhibiting apoptosis, ROS overproduction, and DNA damage. ${ }^{18-20}$ This finding suggests that IGF has an evident neuroprotective effect, which is very important for neurogenesis after nerve injury.

Electrospun nanofibres are among the most commonly used scaffolds and are believed to improve the microenvironment and nerve regeneration after nerve injury by combining therapeutic drugs. The conventional procedure of combining growth factors into polymer materials involves blending growth factors directly into the polymer materials during nanofibre production. However, since the production of polymer materials requires the use of organic solvents, the bioactivity of proteins such as growth factors is decreased or damaged. Recently, some new procedures for the steady immobilisation of growth factors have attracted great interest. Graphene oxide (GO) nanosheets, which are two-dimensional (2D) sheets of $\mathrm{sp}^{2}$-hybridized carbon atoms joined by covalent bonds to form a two-dimensional hexagonal lattice, have garnered significant attention. Because of its superlative physicochemical properties, such as hydrophilic functional groups, low toxicity, exceptional biocompatibility and large loading capacity, GO has great application potential in the field of neuroscience. ${ }^{21} \mathrm{GO}$ could stimulate the differentiation of NSCs into neurons and oligodendrocytes. ${ }^{21,22}$ Neuronal axonal regeneration and orientation have also been improved on the GO substrate, which was useful for building new neural connections. ${ }^{23,24}$ Furthermore, GO is composed of hydrophobic $\pi$ domains in the core region and ionized groups along the edges. These characteristics enhance its binding affinity with proteins via hydrophobic and electrostatic interactions. A recent study showed that graphene-polymer composites efficiently adsorb the growth factor BMP-2 and repair bone defects. ${ }^{25}$ Therefore, GO can not only deliver growth factors but also protect their biological activity. The electrical properties of GO are also beneficial to NSC neuronal differentiation and axonal regeneration.

Thus, in this study, we reported graphene oxideincorporated PLGA nanofibre membranes with immobilised IGF to enhance NSC bioactivity. The PLGA/GO nanofibre membrane was produced by an emulsion-solvent evaporation method, and IGF-1 was immobilised on the surface of the membrane by immersing the biomaterial in solutions of IGF-1 with different concentrations. The survival, proliferation, and differentiation of NSCs on the nanofibres were investigated. This study not only provided a potential biomaterial for nerve repair but also contributed to improving the understanding of the biological applications of engineered graphene-based nanomaterials.

\section{Materials and methods}

\subsection{Materials}

Poly(lactide-co-glycolide) (PLGA, $M_{\mathrm{w}}=70000 \mathrm{~g} \mathrm{~mol}^{-1}$ ) with a lactic acid-glycolic acid ratio of $80: 20$ was purchased from Changchun Sino Biomaterials Co., Ltd, China. IGF-1 was purchased from PeproTech (USA). GO was purchased from Chengdu Organic Chemicals Co., Ltd., China (thickness: 0.55$1.2 \mathrm{~nm}$; diameter: 0.5-3 $\mu \mathrm{m})$. 3-(4,5-Dimethyl-2-thiazolyl)-2,5- diphenyl-2- $H$-tetrazolium bromide (MTT) and 1,1,1,3,3,3-hexafluoroisopropanol (HFIP) were purchased from Sigma-Aldrich (USA). Cetylpyridinium chloride (CPC) was purchased from Aladdin Chemistry Co., Ltd, China. NSC culture media components were purchased from Gibco (USA). Reagents for cell experiments were purchased from Gibco (USA).

\subsection{Fabrication of graphene oxide-incorporated PLGA electrospun nanofibres}

Graphene oxide-incorporated PLGA electrospun nanofibres were fabricated by electrospinning. Briefly, $10 \mathrm{mg}$ GO was added into $1 \mathrm{ml}$ HFIP to prepare a GO suspension, and $190 \mathrm{mg}$ PLGA was dissolved in $9 \mathrm{ml}$ HFIP to prepare a PLGA solution. Then, the GO suspension was dropped into the PLGA solution, and the resulting solution was gently stirred overnight at room temperature to obtain a homogenous suspension. PLGA without the addition of GO served as a control group. An electrospinning setup was employed to convert the mixed solution to mats. The electrospinning parameters were as follows: applied voltage: $40 \mathrm{kV}$; air gap distance: $20 \mathrm{~cm}$; inner diameter of spinneret: $0.4 \mathrm{~mm}$; flow rate of the solution: $0.07-0.10$ $\mathrm{ml} \mathrm{min}^{-1}$. The collected mats were dried in vacuum at room temperature for approximately $48 \mathrm{~h}$ to remove the residual solvent.

\subsection{Characterization of the electrospun nanofibres}

The surface morphology and topography of the nanofibres were examined by a scanning electron microscope (SEM, XL 30 ESEM-FEG, FEI). X-ray diffraction (XRD, D8 ADVANCE, Germany) was employed to determine the elemental composition of the electrospun nanofibres. A static water contact angle was used to evaluate the surface wettability of the nanofibres using the sessile drop method on a contact angle system (VCA 2000, AST).

\subsection{Immobilisation and determination of IGF-1 on the electrospun nanofibres}

The graphene oxide-incorporated PLGA and PLGA electrospun nanofibres were cut to a suitable size and placed in a 24 -well plate. IGF-1 $\left(10,100\right.$, and $\left.500 \mathrm{ng} \mathrm{ml}^{-1}\right)$ in phosphate buffer saline (PBS) was added to each well for $4 \mathrm{~h}$ with continuous shaking by an oscillator at room temperature. The nanofibres were then washed with distilled water three times and stored for further study. The binding efficiency and release profiles of IGF1 were identified by an enzyme-linked immunosorbent assay (ELISA). One millilitre of IGF-1 (500 $\mathrm{ng} \mathrm{ml}^{-1}$ ) was added to a 24well plate. The supernatants were collected, and then IGF-1immobilised nanofibres were washed with PBS three times. The washing liquid was collected and mixed with the previously collected supernatants. The amount of IGF-1 in the obtained combined solution was evaluated with an ELISA kit according to the manufacturer's instructions using a microplate reader (Tecan Infinite M200). All the experiments were performed in triplicate, and the quantitative values were expressed as the average \pm standard deviation. 
Table 1 List of genes and primer nucleotide sequences

\begin{tabular}{ll}
\hline Gene & Primer sequence $\left(5^{\prime}-3^{\prime}\right)$ \\
\hline \multirow{2}{*}{ Nestin } & F AAAGTTCCAGCTGGCTGTGG; \\
TUJ-1 & R TCCAGCTTGGGGTCCTGAAA \\
& F GATCGGAGCCAAGTTCTG; \\
GFAP & R GTCCATCGTCCCAGGTTC \\
& F GCAGACCTTCTCCAACCTG; \\
GAPDH & R ACTCCTTAATGACCTCTCCATC \\
& F TCGCCAGCCGAGCCA; \\
& R CCTTGACGGTGCCATGGAT
\end{tabular}

\subsection{Neural stems cells culture}

Neural stem cells were isolated from the cerebral cortex of embryonic mice (E11.5) as previously described. ${ }^{26}$ Dissociated cells were cultured in defined medium, purified at a density of 50000 cells per $\mathrm{cm}^{2}$ in T25 culture flasks (Corning, NY, USA) and incubated at $37^{\circ} \mathrm{C}$ in a $5 \% \mathrm{CO}_{2}$ humidified incubator. The culture media was changed every other day, and the cells were passaged when the size of the largest neurosphere exceeded 300 $\mu \mathrm{m}$ in diameter. Adherent cells were discarded to obtain purified neural stem cells, and suspended neurospheres were collected by centrifugation, mechanically dissociated as single cells in a new T25 culture flask at a density of 50000 cells per $\mathrm{cm}^{2}$ and prepared for further study.

\subsection{Cell survival assays}

A CCK-8 assay was used to determine NSC cell viability according to the manufacturer's instructions. NSCs cultured on different nanofibres were exposed to $100 \mu \mathrm{M} \mathrm{H}_{2} \mathrm{O}_{2}$ for $24 \mathrm{~h}$.
Then, the existing medium in every well was removed and replaced with $110 \mu \mathrm{l}$ of CCK-8 solution $(10 \mu \mathrm{l}$ CCK- 8 reagent and $100 \mu \mathrm{l}$ DMEM with supplements), followed by incubation at $37^{\circ} \mathrm{C}$ for $3 \mathrm{~h}$. The supernatants were transferred to a new $96-w e l l$ plate after incubation, and the absorbance of the coloured solution was measured using a microplate reader at a wavelength of $450 \mathrm{~nm}$ (the maximum absorption wavelength of formazan).

\subsection{Cell proliferation assays}

Cell proliferation was assessed by an MTT assay at 1, 3 and 7 days. Before cell seeding, the nanofibres were placed in a culture plate and incubated in $2 \mathrm{ml}$ of $10 \mathrm{ng} \mathrm{ml}^{-1}$ poly-D-lysine (PDL, Sigma, USA) solution overnight in an incubator to ensure cell adhesion. Then, NSCs were seeded at a density of 30000 cells per $\mathrm{cm}^{3}$ and cultured in defined medium. Then, $100 \mu \mathrm{l}$ of MTT (Sigma) stock solution in PBS ( $5 \mathrm{mg} \mathrm{ml}^{-1}$ ) was added to each well, and the cells were incubated at $37^{\circ} \mathrm{C}$ for an additional $4 \mathrm{~h}$. Then, the medium was removed, and $750 \mu \mathrm{l}$ of acidified isopropanol $(2 \mathrm{ml}$ of $0.04 \mathrm{~N}$ hydrochloric acid $(\mathrm{HCl})$ in $100 \mathrm{ml}$ of isopropanol) was added to each well to dissolve the formazan crystals. The optical density (OD) was measured at a wavelength of $540 \mathrm{~nm}$ on a fullwavelength microplate reader (Infinite M200, TECAN).

\subsection{Immunofluorescence staining}

To identify and evaluate the differentiation of NSCs, immunofluorescence staining was performed. The culture medium was discarded, and the cells were rinsed with PBS and fixed with $4 \%$ formaldehyde for $30 \mathrm{~min}$. Triton X-100 solution and normal goat serum were used as permeabilization and blocking agents,
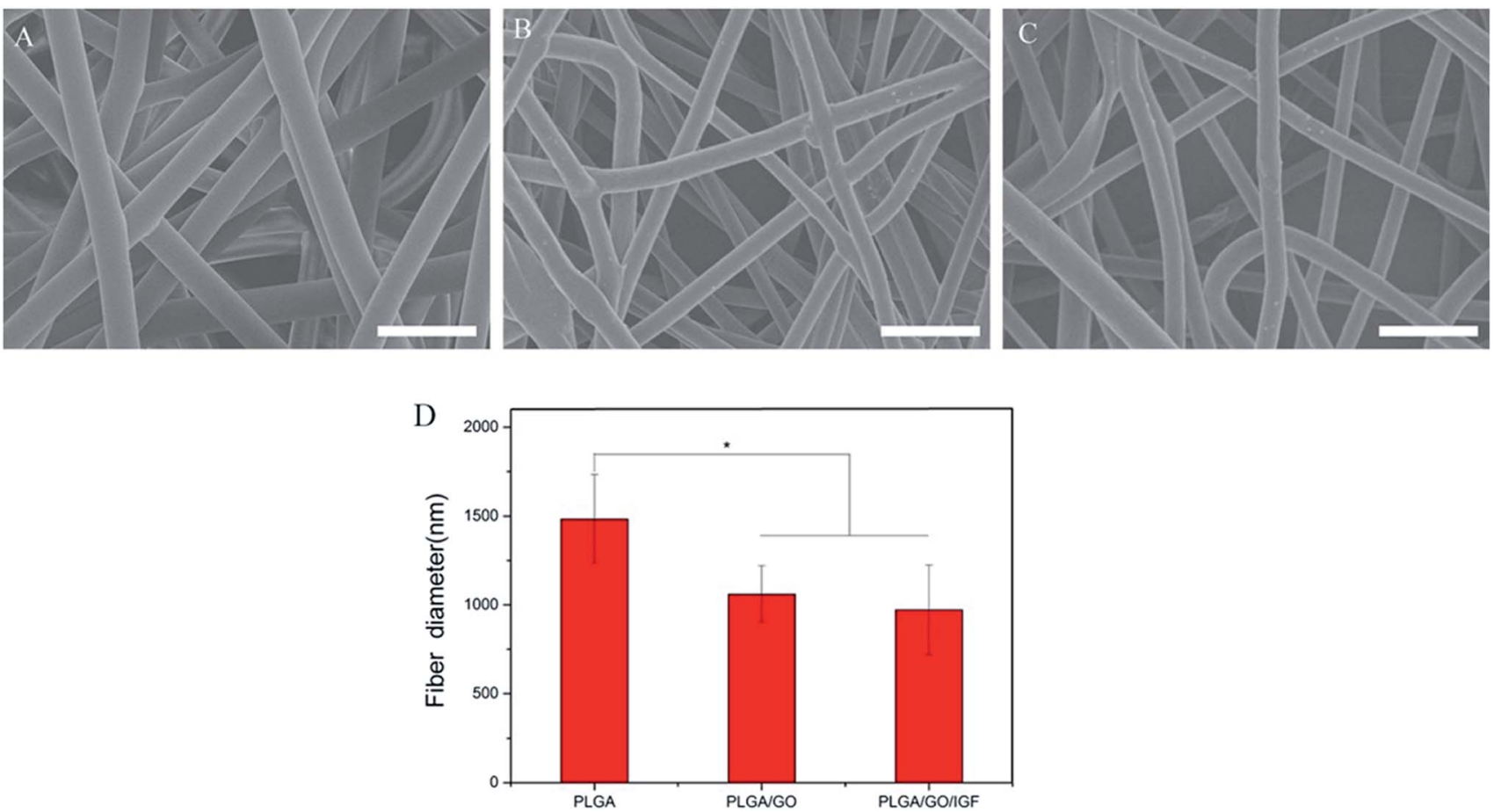

Fig. 1 SEM images of PLGA (A), PLGA/GO (B) and PLGA/GO/IGF (C) nanofibres. Bar lengths are $5 \mu \mathrm{m}$. (D) The fibre diameter of the nanofibres. $* p<0.05 ; n=4$. 
A

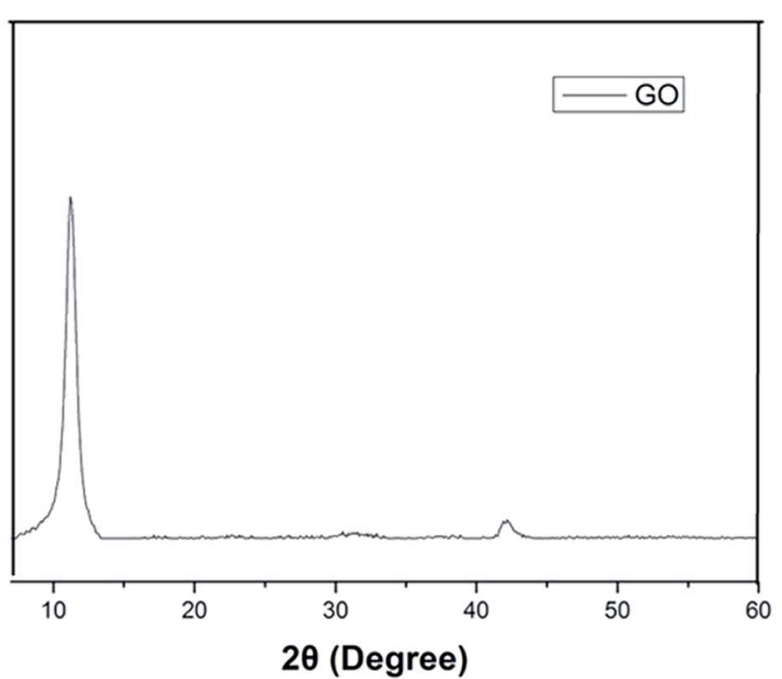

B

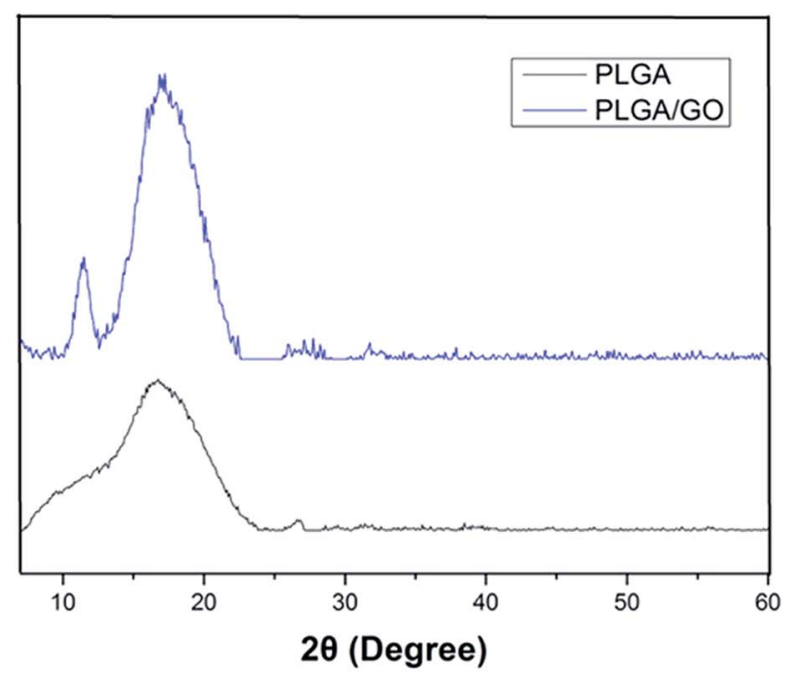

Fig. 2 XRD patterns of GO (A) and the electrospun nanofibres (B).

respectively. Then, primary antibodies, including Nestin as a marker for NSCs (1: 150 dilution; Santa Cruz, Germany), TUJ1 as a marker for neurons ( $1: 100$ dilution; Millipore, Germany), and GFAP as a marker for astrocytic differentiation of NSCs ( $1: 150$ dilution, Millipore, Germany), were used. Subsequently, the samples were washed with PBS three times, followed by incubation in fluorescein isothiocyanate (FITC)-labelled secondary antibodies (1:500 dilution, Abcam) under ambient temperature in the dark for $60 \mathrm{~min}$. Finally, cell nuclei were stained with 4',6-diamidino-2-phenylindole (DAPI) for $1 \mathrm{~min}$. Images were acquired on a confocal laser scanning microscope (LSM 780, ZEISS).

\subsection{Quantitative real-time PCR analysis}

NSC differentiation on electrospun nanofibres was assessed by gene expression using real-time polymerase chain reaction (RTPCR) on day 7. The total RNA concentration and purity were detected by a Nanodrop system (Tecan M200). First-strand cDNA was synthesized by reverse transcriptase as described in the M-MLV manual (Promega). Gene-specific primers were designed using Beacon 5.0 primer design software. The specificity of oligonucleotides was verified by BLASTN ${ }^{\circledR}$ (Basic Local Alignment Search Tool) against the mouse RefSeq RNA database at NCBI. All samples were analysed in triplicate in 8-striped optical tubes (Axygen) using a qPCR SYBR Green Mix Kit (Stratagene). PCR amplification was performed as follows: initial heating at $95{ }^{\circ} \mathrm{C}$ for $10 \mathrm{~min}$, followed by 40 cycles at $95{ }^{\circ} \mathrm{C}$ for $30 \mathrm{~s}, 58{ }^{\circ} \mathrm{C}$ for $60 \mathrm{~s}$, and $72{ }^{\circ} \mathrm{C}$ for $60 \mathrm{~s}$. Each gene expression value was normalized to that of the housekeeping gene glyceraldehyde-3-phosphate dehydrogenase (GAPDH). The results were reported as the relative gene expression. The genes and primer information is shown in Table 1.

\subsection{Statistical analysis}

All quantitative data were analysed with OriginPro 8.0 software (Origin Lab Corporation, USA) and presented as the mean \pm standard deviation. Statistical differences were analysed by oneway analysis of variance (ANOVA). A $p$-value of $p<0.05$ was considered statistically significant.

\section{Results and discussion}

\subsection{Surface characterization of electrospun nanofibres}

The microstructure and the average fibre diameter of the PLGA, PLGA/GO and PLGA/GO/IGF-1 (100 $\mathrm{ng} \mathrm{ml}^{-1}$ ) electrospun nanofibres were observed by SEM. As shown in Fig. 1A, pure PLGA fibres with diameters of $1483.5 \pm 248.6 \mathrm{~nm}$ were formed with a random orientation. There was a significant decrease in the electrospun fibre diameter for the PLGA/GO and PLGA/GO/ IGF nanofibres compared to the pure PLGA nanofibres $(p<0.05$, Fig. 1D). The fibre diameters of the PLGA/GO and PLGA/GO/IGF nanofibres were $1060.2 \pm 159.6$ and $971.8 \pm 252.5 \mathrm{~nm}$, respectively, without significant differences between these two groups $(p>0.05$, Fig. 1D). The decrease in the fibre diameter was likely due to the change in the solution conductivity and viscosity

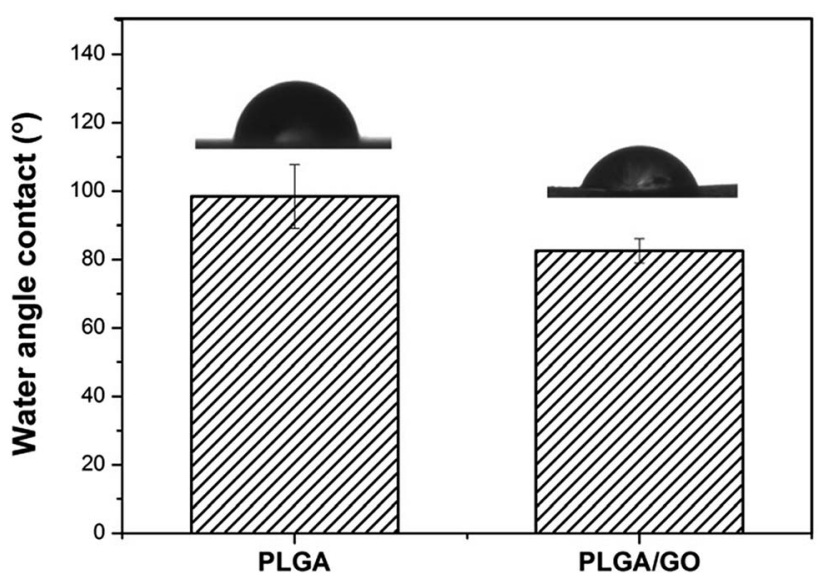

Fig. 3 Water contact angles of pure PLGA nanofibres (A) and PLGA/ GO nanofibres (B). 

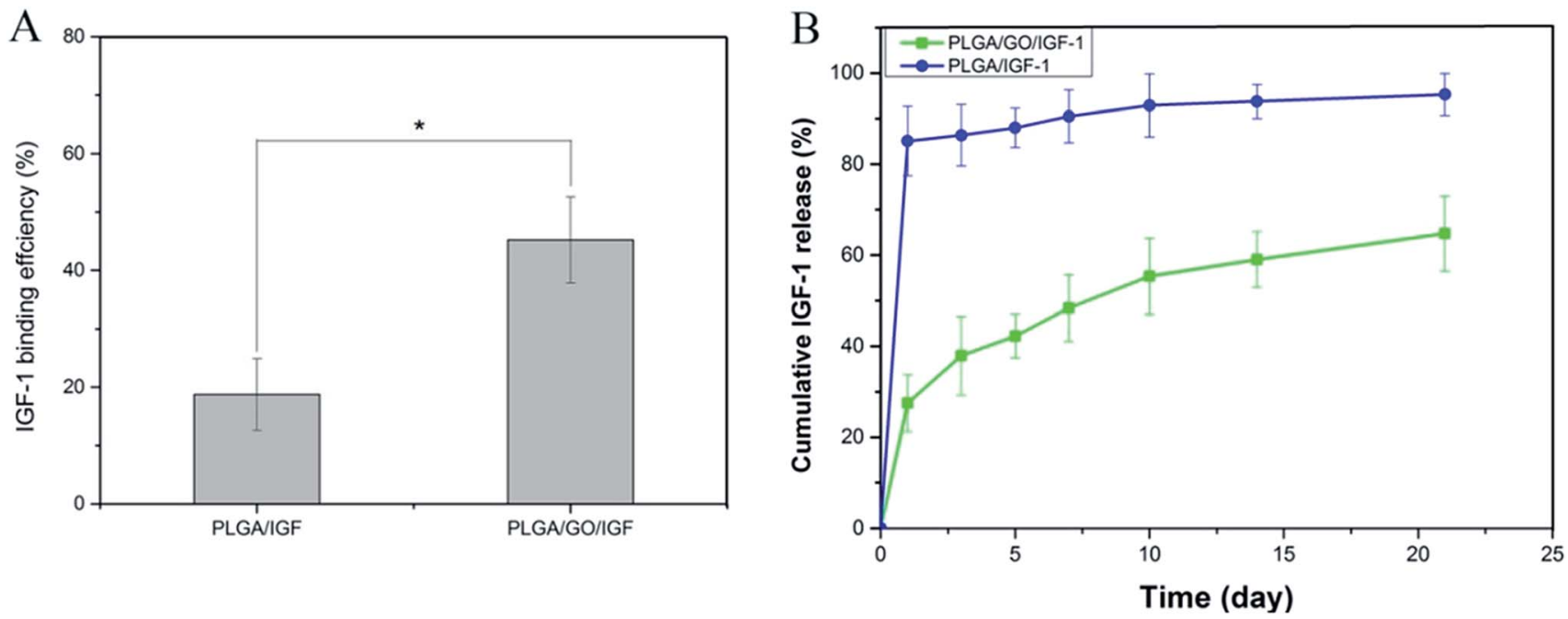

Fig. 4 (A) Binding efficiency of IGF-1 to PLGA and PLGA/GO electrospun nanofibres. (B) IGF-1 release profile from PLGA and PLGA/GO electrospun nanofibres. $* p<0.05 ; n=3$.

caused by the GO. The porous structure and diameter of the nanofibrous matrices were similar to the topological structure and therefore could be favourable for nutrient-waste exchange and cell growth. The XRD patterns of GO and the electrospun nanofibres are shown in Fig. 2. The GO pattern showed a characteristic peak at $2 \theta \approx 12^{\circ}$, and the interlayer spacing was approximately $0.80 \mathrm{~nm}$, which is the typical separation of layered GO. The XRD patterns of the PLGA/GO nanofibres exhibited a similar diffraction peak at $2 \theta \approx 12^{\circ}$. These results suggested that $\mathrm{GO}$ was exposed on the surface of the nanofibres.

\subsection{Contact angle analysis}

The hydrophilicity of materials plays an essential role in cell interactions. Hydrophilic surfaces, which improve growth factor delivery across the interface of biomaterials containing biological factors, are better than hydrophobic surfaces for the adsorption of proteins, such as fibronectin, vitronectin and various growth factors. In our study, the contact angle of pure PLGA and PLGA/GO was measured to analyse the change in the hydrophilicity of the nanofibre surface. As shown in Fig. 3, the contact angles of the pure PLGA nanofibres and the PLGA/GO

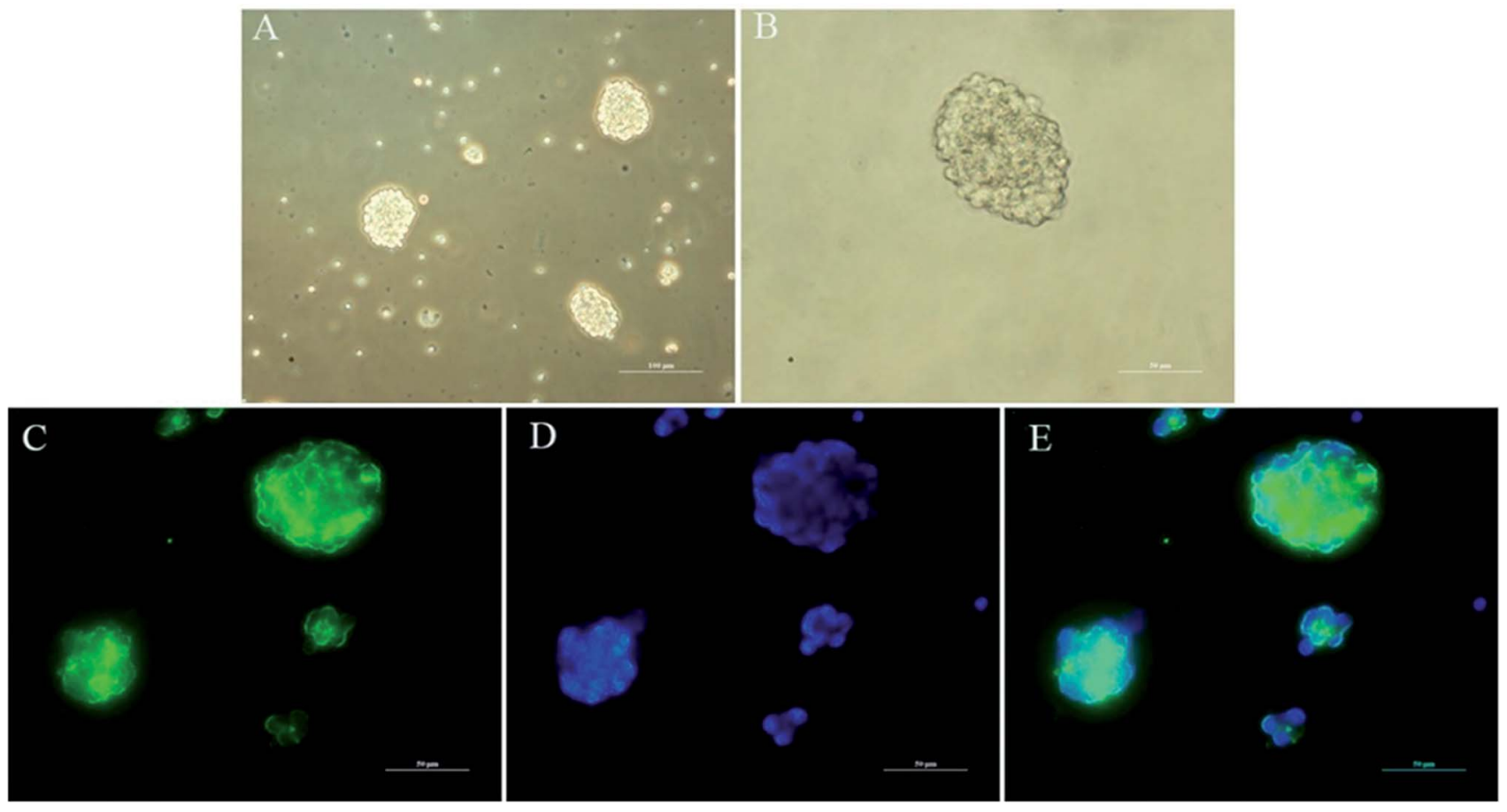

Fig. 5 Identification of primary cultured NSCs in vitro. (A) Representative neurospheres in culture. Scale bar $=100 \mu \mathrm{m}$. (B) Representative neurospheres in culture. Scale bar $=50 \mu \mathrm{m}$. (C-E) Immunocytochemical staining of purified NSCs. Immunostaining makers included Nestin (C, green) for NSCs and DAPI (D, blue) for nuclei, and their images are merged (E). Scale bar $=50 \mu \mathrm{m}$. 

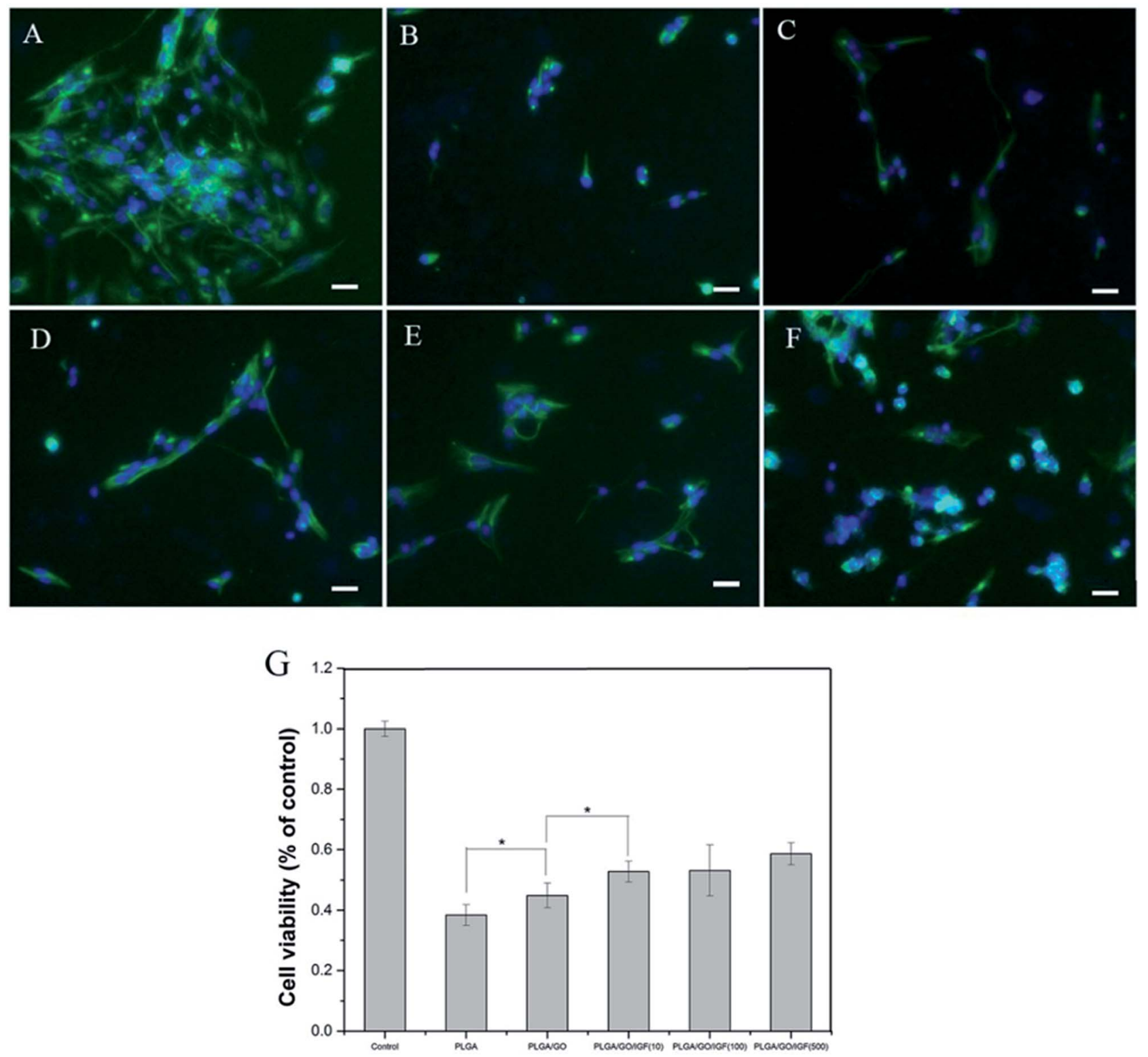

Fig. 6 Cell morphology and viability of NSCs after pre-treatment with $\mathrm{H}_{2} \mathrm{O}_{2}$ in vitro. Immunostaining of NSCs on control (A), PLGA (B), PLGA/GO (C), and PLGA/GO/IGF-1 $\left(10,100,500 \mathrm{ng} \mathrm{m}^{-1}\right)$ samples (D-F) after $24 \mathrm{~h}$. Nestin for NSCs is shown in green, while DAPI for cell nuclei is shown in blue. All scale bar lengths are $100 \mu \mathrm{m}$. (G) The cell survival ratio was measured by a CCK-8 assay after pre-treatment with $\mathrm{H}_{2} \mathrm{O}_{2}$ in vitro. $* p<0.05$; $n=4$.

nanofibres were $98.47 \pm 9.28^{\circ}$ and $82.6 \pm 3.56^{\circ}$, respectively. The results of the contact angle measurements demonstrated that the hydrophilicity of PLGA was improved due to the blending of the substrates with GO. These results indicated that the PLGA/GO nanofibres had better hydrophilicity than the pure PLGA nanofibres, which can be beneficial for cell adhesion and growth factor delivery.

\subsection{Adsorption and release of IGF-1}

The binding efficiency and release profile of IGF-1 on the PLGA and PLGA/GO nanofibres were identified by ELISA. As shown in Fig. 4, the IGF-1 binding efficiency in the PLGA/GO/IGF group was significantly greater than that in the PLGA/IGF group ( $p<$
0.05 , Fig. 4A). This result indicated that the higher IGF-1 adsorption capacities of the PLGA/GO nanofibres compared to pure PLGA nanofibres were the result of GO incorporation. Furthermore, the IGF-1 release profile showed that there was a burst release, with $42 \%$ of the total amount of immobilised IGF-1 released from the PLGA/GO nanofibres within 5 days. The release subsequently slowed down, with approximately $65 \%$ of the total amount of immobilised IGF-1 released after 21 days. However, for the pure PLGA nanofibres, a burst release with $85 \%$ IGF-1 release was found on the first day, and 95\% IGF-1 was released after 21 days.

Previous studies have already suggested that GO has a strong ability to adsorb various proteins, including cytochrome c, bovine serum albumin, ribonuclease $\mathrm{A}$, and protein kinase 
A. ${ }^{27,28}$ We speculated that the mechanism of IGF-1 binding to GO is effectively due to the unique properties of GO. First, the $\pi$ electron cloud of GO is able to interact with the hydrophobic core of proteins. ${ }^{29}$ Additionally, because of $\mathrm{H}$-bonding and electrostatic interactions, GO allowed the non-covalent binding of proteins as well. ${ }^{30}$ In addition, based on our results from the contact angle analysis, GO creates a hydrophilic surface on nanofibres, which could be favourable for more proteins to be immobilised on the surface. ${ }^{31}$ Furthermore, PLGA/GO effectively reduced the burst release of IGF-1 within 5 days and maintained a sustained stable IGF-1 release rate, resulting in the long-term bioactivity of the composites for neurogenesis. The abovementioned results suggested that the PLGA/GO nanofibres have good affinity for IGF-1 and are regarded as a useful carrier for IGF-1 delivery.

\subsection{Primary cultured NSC identification}

The ability to clonally form neurospheres is an indicator of selfrenewal. Single NSCs assembled together to create neurospheres spontaneously after 3 days of suspension culture (Fig. 5A and B). We performed immunocytochemistry with antiNestin to identify NSCs. The results indicated that the neurospheres were positive for Nestin and could be used for subsequent studies.

\subsection{NSC survival rate}

Nerve injury can result in the overproduction of reactive oxygen species (ROS) and inflammation, which produce a catastrophic microenvironment for NSC survival and inhibit nerve regeneration. Designing a new scaffold with a neuroprotective effect to improve NSC survival is considered to have great potential for therapy. In our study, $\mathrm{H}_{2} \mathrm{O}_{2}$ was used to simulate the microenvironment after nerve injury, and a CCK-8 assay was performed to evaluate NSC survival in different nanofibres. As shown in Fig. 6G, the cell viability after pre-treatment with $\mathrm{H}_{2} \mathrm{O}_{2}$ was significantly lower compared to the control group. The cell survival rate increased dramatically in the PLGA/GO and PLGA/ GO/IGF (at all three concentrations) groups compared to the PLGA group. A dramatically higher survival rate in the PLGA/ GO/IGF (at all three concentrations) groups compared to the PLGA/GO group was found. Immunofluorescence staining of Nestin was evaluated to better observe NSC morphology in the inflammatory microenvironment. As shown in Fig. 6A-F, NSCs assembled and exhibited a high degree of spreading with a fusiform shape in the control group, while sporadic NSCs with an abnormal shape were observed in all the groups under $\mathrm{H}_{2} \mathrm{O}_{2}$ pre-treatment. Although IGF-1 immobilisation could significantly improve the NSC survival rate, the NSC morphology still showed a pathologic change.

$\mathrm{H}_{2} \mathrm{O}_{2}$ in medium mediates oxidative injury and inflammation in vitro, thus simulating the tissue microenvironment after nerve injury. ${ }^{32-34}$ Our results showed that NSCs on the PLGA/GO nanofibres had a better survival rate than PLGA alone, suggesting that GO was effective in preventing NSCs on the surface of the graphene-polymer hybrid scaffolds from reactive oxygen species (ROS)-mediated cell death. Previous research has demonstrated that GO protects mesenchymal stem cells (MSCs) and provides microenvironments suitable for long-term MSC survival in vivo and in vitro. ${ }^{35}$ Our results indicated that GO incorporation on the PLGA nanofibres had a neuroprotective effect on NSC survival under harsh conditions. Moreover, the addition of IGF-1 in the PLGA/GO nanofibres further significantly increased the NSC survival rate. Evidence has indicated that IGF-I improves the survival of multiple types of nerve cells, such as NSCs, Purkinje cells, and motoneurons. ${ }^{18,36,37}$ A lack of IGF-I or IGF-IR causes a decrease in neuronal survival following hypoxic/ischaemic injuries in the brain, whereas IGF-I overexpression increases neuronal survival in the lesioned site in the brain. ${ }^{19,20}$ In addition, IGF-I could prevent neuronal death in neurodegenerative diseases by regulating the accumulation of amyloid-b and Tau proteins. ${ }^{38}$ Based on our results, we believe that IGF-I has a strong neuroprotective effect on NSC survival in a microenvironment with oxidative stress and inflammation. Although the mechanism remains unclear, several studies have indicated that the neuroprotective effect of IGF-1 might be related to PI3K/AKT signalling. ${ }^{39,40}$ Since the NSC survival rate was higher when the IGF-1 concentration was 100 and $500 \mathrm{ng}$ $\mathrm{ml}^{-1}$, we suggest that IGF-1 has a dose-dependent neuroprotective function and that a relatively higher dose of IGF-1 immobilised to the nanofibres induces better cell survival.

\subsection{Proliferation of NSCs on the nanofibres}

Endogenous NSC proliferation in the nerve injury site is a key step in neurogenesis, and cell proliferation enhancement on the surface of biomaterials is typically responsible for eventual tissue formation. Accordingly, the NSC proliferation rate is an essential factor to evaluate the therapeutic effect of electrospun nanofibres. The proliferation of NSCs on the different nanofibres (PLGA, PLGA/GO, and PLGA/GO/IGF-1 (10 100500 ng $\mathrm{ml}^{-1}$ )) was assessed using an MTT assay from 1 to 7 days.

As shown in Fig. 7, cell proliferation was observed on all the nanofibres with increasing incubation up to 7 days. We found

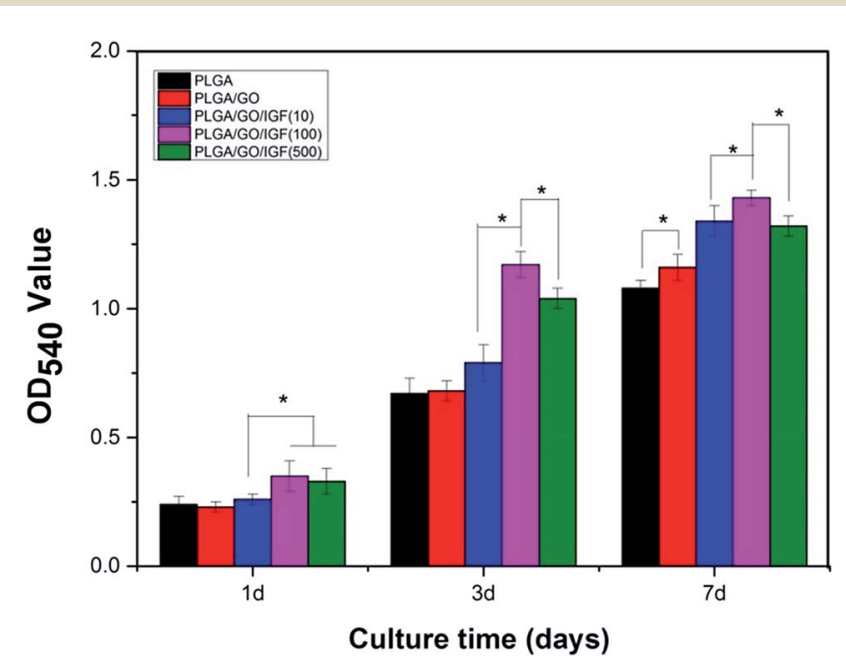

Fig. 7 Proliferation of NSCs cultured on PLGA, PLGA/GO, and PLGA/ GO/IGF-1 $\left(10,100,500 \mathrm{ng} \mathrm{ml}^{-1}\right)$ for 1,3 , and 7 days in vitro. $* p<0.05$; $n=4$. 


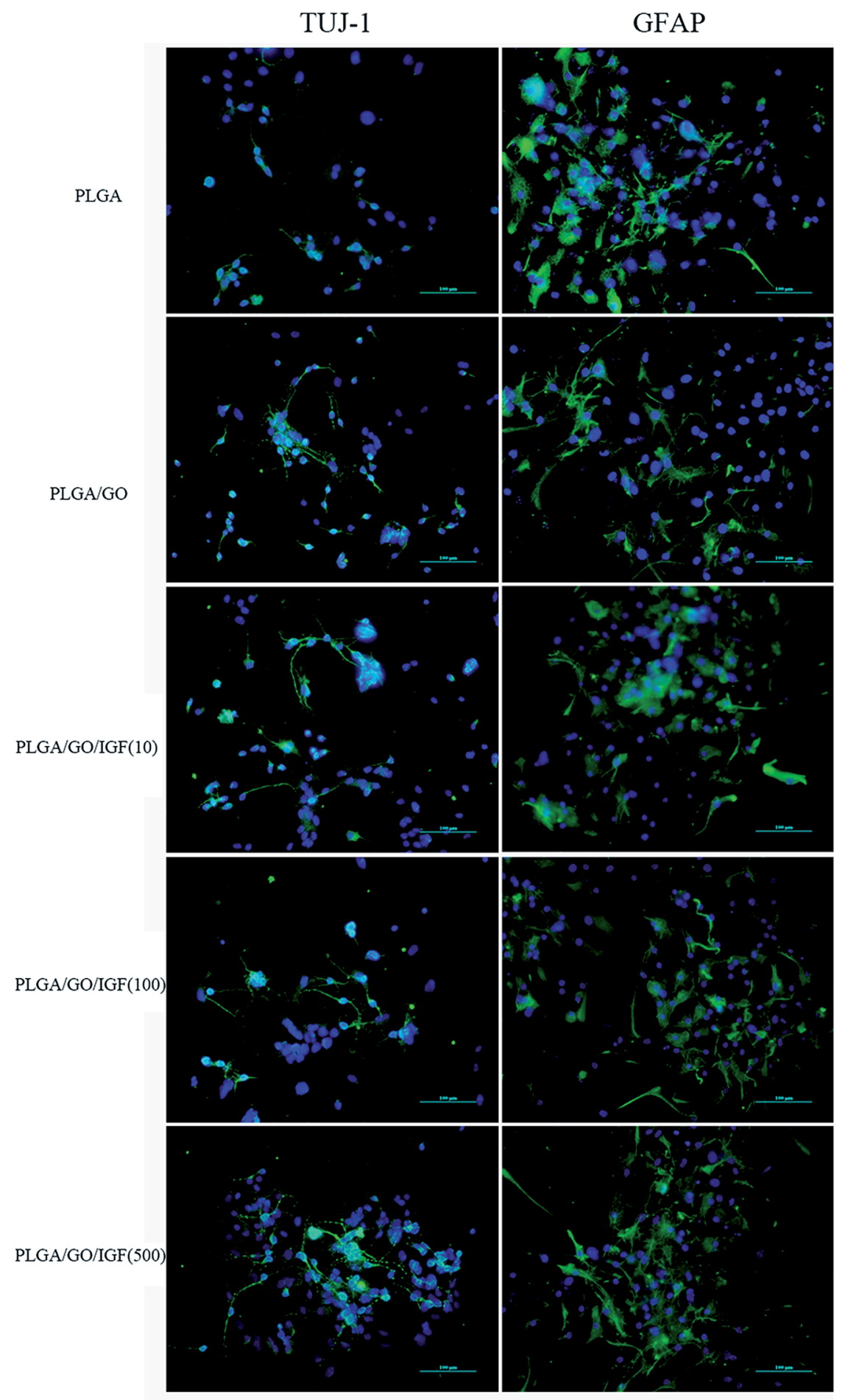

Fig. 8 Immunofluorescence staining was performed in NSCs after 7 days of differentiation. The markers are shown in green, while the cell nuclei, counterstained with DAPI, are shown in blue. All scale bar lengths are $100 \mu \mathrm{m}$.

that the OD value in the PLGA/GO group was dramatically greater than that in the PLGA group at 7 days. Furthermore, the OD value was significantly higher in the three PLGA/GO/IGF-1 groups than in the PLGA and PLGA/GO groups $(p<0.05$, Fig. 7), while $100 \mathrm{ng} \mathrm{ml} \mathrm{m}^{-1}$ IGF-1 was the most effective concentration in the enhancement of NSC proliferation among the three concentrations used in this study, although there was no significant difference between PLGA/GO/IGF-1 (100 ng ml $\mathrm{ml}^{-1}$ ) and PLGA/GO/IGF-1 (500 ng ml ${ }^{-1}$ ) on the first day.

Previous studies have demonstrated that graphene-polymer composites could enhance cell proliferation, which might be attributed to the rapid absorption of proteins due to $\pi-\pi$ stacking between aromatic rings in GO, thus providing a biocompatible environment for cells to adhere and 
A

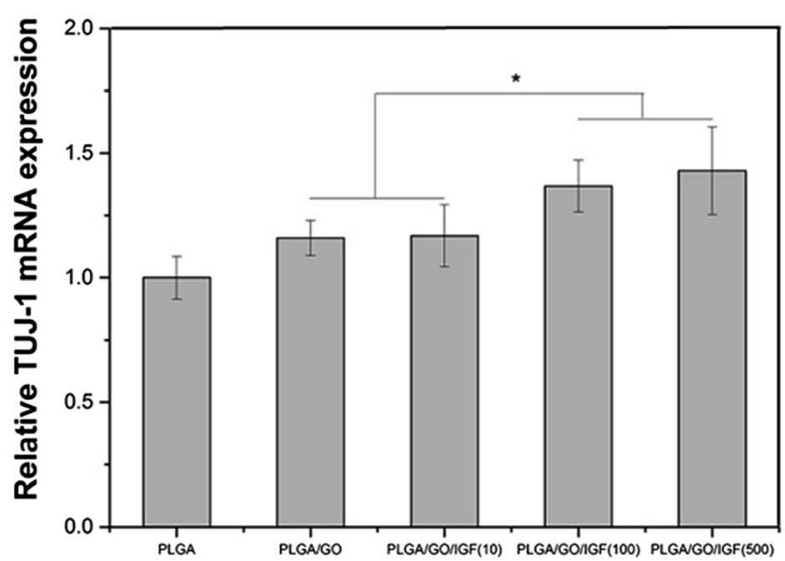

B

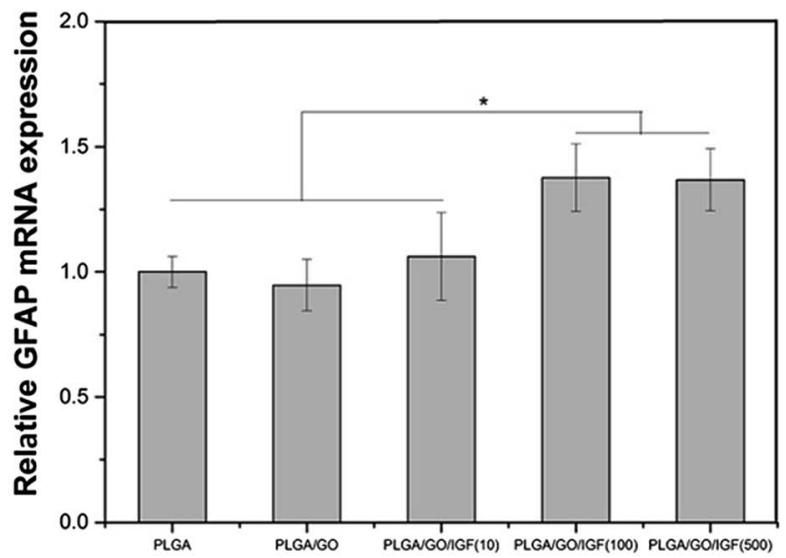

Fig. 9 Quantitative real-time PCR analysis of TUJ-1 (A) and GFAP (B) after NSCs were cultured for 7 days. * $p<0.05 ; n=4$.

proliferate. ${ }^{40}$ Additionally, the hydrophilic surface of the graphene-reinforced polymer composites caused the expression of various integrins and connexins, which also had an impact on cell adhesion and proliferation. ${ }^{41}$ However, we found that the noticeable positive effect of $\mathrm{GO}$ on cell proliferation only occurred at day 7, while GO seemed to have a slight inhibitory effect on cell proliferation at day 1 , which indicated that the positive impact of GO on NSC proliferation requires a specific period for the interaction between $\mathrm{GO}$ and cells. Compared to nanofibres without IGF-1 immobilisation, when IGF-1 was immobilised to the nanofibres, an enhancement in cell proliferation was observed in our study. Among the five groups of nanofibres, PLGA/GO/IGF-1 (100 ng ml ${ }^{-1}$ ) demonstrated the greatest potential in promoting cell proliferation. IGF-1, either alone or in combination with other active ingredients, has garnered interest as a therapeutic factor. IGF-I supports the proliferation of neural cells by interacting with IGF-IR, which may activate the PI3K/AKT and MAP kinase pathways. ${ }^{42}$ Several studies have shown that IGF-I promotes the proliferation of NSCs both in vitro and in vivo. ${ }^{13,43-45}$ In this study, IGF-1 was effectively immobilised onto the PLGA/GO nanofibres, and NSC proliferation increased the most at 1,3 , and 7 days when the concentration of IGF-1 was $100 \mathrm{ng} \mathrm{ml}^{-1}$.

\subsection{Differentiation of NSCs on the nanofibres}

NSCs cultured on the nanofibres for 7 days were analysed by qRT-PCR and immunostaining to evaluate the neural differentiation of NSCs. During NSC differentiation, some of the cells differentiate into neurons, whereas others may differentiate into glial cells, which support nervous tissue and neuronal activity. In our study, TUJ-1 and GFAP were used as markers for neurons and astrocytes, respectively. The mRNA expression level of TUJ-1 in the PLGA/GO group and PLGA/GO/IGF-1 groups (at all three concentrations) was significantly greater than that in the PLGA group (Fig. 9A, $p<0.05$ ). The TUJ-1 expression level gradually increased following IGF-1 delivery, and there was no significant difference between the IGF-1 concentrations of 100 and $500 \mathrm{ng} \mathrm{ml}^{-1}$. A noticeable increase in the mRNA expression level of GFAP was observed in the PLGA/GO/IGF-1 (100 and 500 $\mathrm{ng} \mathrm{m} \mathrm{m}^{-1}$ ) groups compared to the other three groups.
Furthermore, the GFAP gene expression level in the PLGA/GO group was slightly less than that in the PLGA group, and PLGA/GO/IGF-1 (100 ng ml ${ }^{-1}$ ) was not significantly different (Fig. 9B, $p>0.05$ ). To better observe NSC differentiation, the protein expression of TUJ-1 and GFAP was evaluated by immunofluorescence staining. As shown in Fig. 8, after 7 days of culture, most of the TUJ-1-positive cells assembled together with clear axons in the PLGA/GO/IGF-1 (500 $\mathrm{ng} \mathrm{ml}^{-1}$ ) group. Furthermore, TUJ-1 and GFAP expression were higher with higher concentrations (100 and $500 \mathrm{ng} \mathrm{m}^{-1}$ ) of immobilised IGF-1.

Since NSCs have the potential to differentiate into neurons and glial cells, finding effective methods that can progress the NSC differentiation rate is crucial for the treatment of neurological diseases/disorders. The simultaneous dramatic increase in the TUJ-1 expression level and a relatively small decrease in the GFAP expression level were observed in the PLGA/GO group compared to the PLGA group, indicating that the GO-modified PLGA nanofibres promoted selective NSC differentiation. Our study demonstrated that GO had a positive effect on guiding NSCs to differentiate into neurons, which was consistent with previous research. ${ }^{46,47}$ In addition, GO appeared to have a slight inhibition on the differentiation of NSCs towards astrocytes, but the mechanism remained unclear. We speculated that in the PLGA/GO group, GO can be directly deposited on the surface of PLGA, followed by improved mechanical interconnections at the cell-material and cell-cell interfaces, thus being essential for the adhesion, growth, and neuronal differentiation of NSCs. ${ }^{48}$ To achieve better neuroprotective and neurogenesis bioactivity for graphene-polymer scaffolds, we immobilised IGF-1 onto the surface of the nanofibres. After IGF-1 was immobilised to PLGA/GO, all the IGF-1-modified nanofibres showed higher NSC differentiation rates than the PLGA and PLGA/GO nanofibres, suggesting that IGF-1 played an important role in promoting NSC differentiation towards both neurons and astrocytes. Furthermore, we found that the average and high concentrations of IGF-1 could better induce NSC differentiation. However, the ability of IGF-1 to differentiate NSCs was not in the neuronal direction. An evenly improved number of neurons and astrocytes was obtained on the PLGA/ 
GO/IGF nanofibres, indicating that IGF-1 only enhanced the NSC differentiation rate but not the specific differentiated cell type. We observed that when $500 \mathrm{ng} \mathrm{ml}{ }^{-1}$ IGF-1 was immobilised to graphene oxide-incorporated PLGA electrospun nanofibres, the neurons derived from NSCs cultured on these nanofibres were assembled more easily and formed a neuronal network. A previous study demonstrated that IGF-I not only affects NSC proliferation and migration but also is responsible for NSC-directed neuronal positioning and migration. ${ }^{17}$ Therefore, IGF-1 immobilised on the nanofibres causes NSCs to differentiate into neurons, followed by neuronal migration to form a neuronal network.

\section{Conclusion}

In summary, PLGA/GO electrospun nanofibres were successfully fabricated by an emulsion solvent evaporation method. We found that the incorporation of GO effectively enhanced the surface properties of the PLGA/GO nanofibres. Furthermore, the PLGA/GO nanofibres enhanced NSC survival in a microenvironment with oxidative damage and NSC neuronal differentiation to some extent. Additionally, the introduction of GO on the nanofibres clearly increased the binding of IGF-1 to the biomaterial surface and resulted in a sustained IGF-1 release curve. After immobilising IGF-1, the PLGA/GO nanofibres exhibited excellent bioactivities for supporting the survival, proliferation, and differentiation of NSCs. The findings from this study revealed that PLGA/GO might be an effective carrier for IGF-1 delivery, and the immobilisation of IGF-1 onto PLGA/ GO nanofibres has excellent potential for the enhancement of the neuroprotective and neurogenic effects of nerve implants.

\section{Conflicts of interest}

There are no conflicts of interest to declare.

\section{Acknowledgements}

This study was supported by the by the National Natural Science Foundation of China, No. 81672263, No. 31572217 and Graduate Innovation Fund of Jilin University, No. $101832018 \mathrm{C} 074$.

\section{References}

1 S. Liu, X. Sun, T. Wang, et al., Nano-fibrous and ladder-like multi-channel nerve conduits: degradation and modification by gelatin, Mater. Sci. Eng., C, 2018, 83, 130142.

2 K. Meletis, F. Barnabe-Heider, M. Carlen, et al., Spinal cord injury reveals multilineage differentiation of ependymal cells, PLoS Biol., 2008, 6, e182.

3 A. J. Mothe and C. H. Tator, Proliferation, migration, and differentiation of endogenous ependymal region stem/ progenitor cells following minimal spinal cord injury in the adult rat, Neuroscience, 2005, 131, 177-187.
4 H. Sabelstrom, M. Stenudd, P. Reu, et al., Resident neural stem cells restrict tissue damage and neuronal loss after spinal cord injury in mice, Science, 2013, 342, 637-640.

5 H. Duan, X. Li, C. Wang, et al., Functional hyaluronate collagen scaffolds induce NSCs differentiation into functional neurons in repairing the traumatic brain injury, Acta Biomater., 2016, 45, 182-195.

6 B. L. Du, C. G. Zeng, W. Zhang, et al., A comparative study of gelatin sponge scaffolds and PLGA scaffolds transplanted to completely transected spinal cord of rat, J. Biomed. Mater. Res., Part A, 2014, 102, 1715-1725.

7 E. W. C. Chan, D. Bennet, P. Baek, et al., Electrospun Polythiophene Phenylenes for Tissue Engineering, Biomacromolecules, 2018, 19, 1456-1468.

8 S. Kim, J. E. Jang, J. H. Lee, et al., Composite scaffold of micronized porcine cartilage/poly(lacticcoglycolic acid) enhances anti-inflammatory effect, Mater. Sci. Eng., C, 2018, 88, 46-52.

9 G. Jiao, Y. Pan, C. Wang, et al., A bridging SF/Alg composite scaffold loaded NGF for spinal cord injury repair, Mater. Sci. Eng., C, 2017, 76, 81-87.

10 J. Zhang, X. Lu, G. Feng, et al., Chitosan scaffolds induce human dental pulp stem cells to neural differentiation: potential roles for spinal cord injury therapy, Cell Tissue Res., 2016, 366, 129-142.

11 I. Grulova, L. Slovinska, J. Blasko, et al., Delivery of Alginate Scaffold Releasing Two Trophic Factors for Spinal Cord Injury Repair, Sci. Rep., 2015, 5, 13702.

12 A. N. Ziegler, J. S. Schneider, M. Qin, et al., IGF-II promotes stemness of neural restricted precursors, Stem Cells, 2012, 30, 1265-1276.

13 Q. Hu, S. Y. Lee, J. R. O'Kusky, et al., Signalling through the type 1 insulin-like growth factor receptor (IGF1R) interacts with canonical Wnt signalling to promote neural proliferation in developing brain, ASN Neuro, 2012, 4, e00092.

$14 \mathrm{H}$. Yuan, R. Chen, L. Wu, et al., The regulatory mechanism of neurogenesis by IGF-1 in adult mice, Mol. Neurobiol., 2015, 51, 512-522.

15 P. Ye, G. J. Popken, A. Kemper, et al., Astrocyte-specific overexpression of insulin-like growth factor-I promotes brain overgrowth and glial fibrillary acidic protein expression, J. Neurosci. Res., 2004, 78, 472-484.

16 Y. Cao, A. J. Gunn, L. Bennet, et al., Insulin-like growth factor (IGF)-1 suppresses oligodendrocyte caspase-3 activation and increases glial proliferation after ischemia in near-term fetal sheep, J. Cereb. Blood Flow Metab., 2003, 23, 739-747.

17 A. Hurtado-Chong, M. J. Yusta-Boyo, E. Vergano-Vera, et al., IGF-I promotes neuronal migration and positioning in the olfactory bulb and the exit of neuroblasts from the subventricular zone, Eur. J. Neurosci., 2009, 30, 742-755.

18 J. S. Lunn, S. A. Sakowski, L. M. McGinley, et al., Autocrine production of IGF-I increases stem cell-mediated neuroprotection, Stem Cells, 2015, 33, 1480-1489.

19 W. Liu, J. A. D'Ercole and P. Ye, Blunting type 1 insulin-like growth factor receptor expression exacerbates neuronal 
apoptosis following hypoxic/ischemic injury, BMC Neurosci., 2011, 12, 64.

20 S. W. Carlson, S. K. Madathil, D. M. Sama, et al., Conditional overexpression of insulin-like growth factor-1 enhances hippocampal neurogenesis and restores immature neuron dendritic processes after traumatic brain injury, $J$. Neuropathol. Exp. Neurol., 2014, 73, 734-746.

21 E. Garcia-Alegria, M. Iliut, M. Stefanska, et al., Corrigendum: graphene oxide promotes embryonic stem cell differentiation to haematopoietic lineage, Sci. Rep., 2016, 6, 28723.

22 S. Shah, P. T. Yin, T. M. Uehara, et al., Guiding stem cell differentiation into oligodendrocytes using graphenenanofiber hybrid scaffolds, Adv. Mater., 2014, 26, 3673-3680.

23 C. Defterali, R. Verdejo, L. Peponi, et al., Thermally reduced graphene is a permissive material for neurons and astrocytes and de novo neurogenesis in the adult olfactory bulb in vivo, Biomaterials, 2016, 82, 84-93.

24 A. Solanki, S. T. Chueng, P. T. Yin, et al., Axonal alignment and enhanced neuronal differentiation of neural stem cells on graphene-nanoparticle hybrid structures, Adv. Mater., 2013, 25, 5477-5482.

25 C. Fu, X. Yang, S. Tan, et al., Enhancing Cell Proliferation and Osteogenic Differentiation of MC3T3-E1 Preosteoblasts by BMP-2 Delivery in Graphene OxideIncorporated PLGA/HA Biodegradable Microcarriers, Sci. Rep., 2017, 7, 12549.

26 K. Farrell, J. Joshi and C. R. Kothapalli, Injectable uncrosslinked biomimetic hydrogels as candidate scaffolds for neural stem cell delivery, J. Biomed. Mater. Res., Part A, 2017, 105, 790-805.

27 M. Patila, I. V. Pavlidis, A. Kouloumpis, et al., Graphene oxide derivatives with variable alkyl chain length and terminal functional groups as supports for stabilization of cytochrome c, Int. J. Biol. Macromol., 2016, 84, 227-235.

28 H. Shen, M. Liu, H. He, et al., PEGylated graphene oxidemediated protein delivery for cell function regulation, ACS Appl. Mater. Interfaces, 2012, 4, 6317-6323.

29 W. C. Lee, C. H. Lim, H. Shi, et al., Origin of enhanced stem cell growth and differentiation on graphene and graphene oxide, ACS Nano, 2011, 5, 7334-7341.

30 L. D. Quarles, D. A. Yohay, L. W. Lever, et al., Distinct proliferative and differentiated stages of murine MC3T3-E1 cells in culture: an in vitro model of osteoblast development, J. Bone Miner. Res., 1992, 7, 683-692.

31 C. J. Wilson, R. E. Clegg, D. I. Leavesley, et al., Mediation of biomaterial-cell interactions by adsorbed proteins: a review, Tissue Eng., 2005, 11, 1-18.

32 G. Bhatia, V. Dhuna, K. Dhuna, et al., Bacopa monnieri extracts prevent hydrogen peroxide-induced oxidative damage in a cellular model of neuroblastoma IMR32 cells, Chin. J. Nat. Med., 2017, 15, 834-846.

33 X. N. Mao, H. J. Zhou, X. J. Yang, et al., Neuroprotective effect of a novel gastrodin derivative against ischemic brain injury: involvement of peroxiredoxin and TLR4 signaling inhibition, Oncotarget, 2017, 8, 90979-90995.
34 N. Sawamura, M. Yamada, M. Fujiwara, et al., The Neuroprotective Effect of Thalidomide against Ischemia through the Cereblon-mediated Repression of AMPK Activity, Sci. Rep., 2018, 8, 2459.

35 T. H. Kim, T. Lee, W. A. El-Said, et al., Graphene-Based Materials for Stem Cell Applications, Materials, 2015, 8, 8674-8690.

36 M. R. Aburto, M. Magarinos, Y. Leon, et al., AKT signaling mediates IGF-I survival actions on otic neural progenitors, PLoS One, 2012, 7, e30790.

37 L. Croci, V. Barili, D. Chia, et al., Local insulin-like growth factor I expression is essential for Purkinje neuron survival at birth, Cell Death Differ., 2011, 18, 48-59.

38 E. Carro, J. L. Trejo, T. Gomez-Isla, et al., Serum insulin-like growth factor I regulates brain amyloid-beta levels, Nat. Med., 2002, 8, 1390-1397.

39 G. Mairet-Coello, A. Tury and E. DiCicco-Bloom, Insulin-like growth factor-1 promotes $\mathrm{G}(1) / \mathrm{S}$ cell cycle progression through bidirectional regulation of cyclins and cyclindependent kinase inhibitors via the phosphatidylinositol 3kinase/Akt pathway in developing rat cerebral cortex, $J$. Neurosci., 2009, 29, 775-788.

40 S. Kumar and K. Chatterjee, Strontium eluting graphene hybrid nanoparticles augment osteogenesis in a 3D tissue scaffold, Nanoscale, 2015, 7, 2023-2033.

41 D. Mohammadrezaei, H. Golzar, M. Rezai Rad, et al., In vitro effect of graphene structures as an osteoinductive factor in bone tissue engineering: a systematic review, J. Biomed. Mater. Res., Part A, 2018, 2284-2343.

42 G. Otaegi, M. J. Yusta-Boyo, E. Vergano-Vera, et al., Modulation of the PI 3-kinase-Akt signalling pathway by IGF-I and PTEN regulates the differentiation of neural stem/precursor cells, J. Cell Sci., 2006, 119, 2739-2748.

43 M. Magarinos, M. R. Aburto, H. Sanchez-Calderon, et al., RAF kinase activity regulates neuroepithelial cell proliferation and neuronal progenitor cell differentiation during early inner ear development, PLoS One, 2010, 5, e14435.

44 C. Vicario-Abejon, M. J. Yusta-Boyo, C. Fernandez-Moreno, et al., Locally born olfactory bulb stem cells proliferate in response to insulin-related factors and require endogenous insulin-like growth factor-I for differentiation into neurons and glia, J. Neurosci., 2003, 23, 895-906.

$45 \mathrm{P}$. Ye and A. J. D'Ercole, Insulin-like growth factor actions during development of neural stem cells and progenitors in the central nervous system, J. Neurosci. Res., 2006, 83, 1-6.

46 S. Y. Park, J. Park, S. H. Sim, et al., Enhanced differentiation of human neural stem cells into neurons on graphene, $A d v$. Mater., 2011, 23, H263-H267.

47 D. Yang, T. Li, M. Xu, et al., Graphene oxide promotes the differentiation of mouse embryonic stem cells to dopamine neurons, Nanomedicine, 2014, 9, 2445-2455.

48 P. Viswanathan, S. Chirasatitsin, K. Ngamkham, et al., Cell instructive microporous scaffolds through interface engineering, J. Am. Chem. Soc., 2012, 134, 20103-20109. 